\title{
Effect of direct ovarian injection of vascular endothelial growth factor gene fragments on follicular development in immature female rats
}

\author{
Takashi Shimizu $^{1,2}$, Koji lijima², Kanako Miyabayashi ${ }^{2}$, Yoshinori Ogawa $^{2}$, Hitoshi Miyazaki ${ }^{3}$, \\ Hiroshi Sasada ${ }^{2}$ and Eimei Sato ${ }^{2}$ \\ ${ }^{1}$ Graduate School of Animal and Food Hygiene, Obihiro University of Agriculture and Veterinary Medicine, Obihiro, \\ 080-8555, Japan, ${ }^{2}$ Laboratory of Animal Reproduction, Graduate School of Agricultural Science, Tohoku University, \\ 1-1 Tsutsumidori-amamiyamachi, Aoba-ku, Sendai 981-8555, Japan and ${ }^{3}$ Gene Research Center, University of \\ Tsukuba, Tsukuba, Ibaraki 305-8572, Japan
}

Correspondence should be addressed to T Shimizu; Email: shimizut@obihiro.ac.jp

\begin{abstract}
Vascular endothelial growth factor (VEGF) expression in granulosa cells is associated with the thecal vasculature growth during ovarian follicular development. We hypothesized that injection of VEGF gene fragments directly into the rat ovary would induce production of a large number of ovulatory follicles and that these follicles would ovulate. To test this hypothesis, we treated immature female rats with combinations of hormones and VEGF gene fragments. The animals were divided into two groups: one group received solution containing transfection reagents as a control $(n=5)$, while the other group received direct ovarian injection of VEGF gene fragments at 19 ( $n=5), 21$ $(n=5), 23(n=5)$, or $25(n=5)$ days after birth followed by i.p. administration of 20 IU equine chorionic gonadotropin (eCG) at the age of 26 days. Forty-eight hours after eCG injection, animals were given 20 IU human chorionic gonadotropin (hCG) i.p. and then the oocytes in both groups were counted. The maximum number of ovulated oocytes was obtained when the VEGF gene fragments were injected into the rat ovary at 21 days after birth. Histological examination revealed that the injection of VEGF gene fragments markedly increased the vascular density around the preovulatory follicles and also the number of these follicles. Our data provide the first reported evidence that most ovulatory follicles generated by injection of VEGF gene fragments are able to ovulate upon hCG treatment. These results demonstrate that injection of VEGF gene fragments directly into the ovary stimulates the development of antral follicles by inducing the formation of thecal vasculature in immature female rats.

Reproduction (2007) 134 677-682
\end{abstract}

\section{Introduction}

Vascular endothelial growth factor (VEGF) is a heparinbinding growth factor that is specific for vascular endothelial cells and is able to induce angiogenesis in vivo (Leung et al. 1989). In the ovary, VEGF immunoreactivity in granulosa cells is weak or absent from the primordial to multilayered stages of primary follicles, but increases gradually when follicles differentiate to secondary and then to antral follicles (Celik-Ozenci et al. 2003, Greenaway et al. 2005). VEGF expression in granulosa cells (Barboni et al. 2000, Mattioli et al. 2001) is associated with the thecal vasculature growth during ovarian follicular development (Shimizu et al. 2002).

The local administration of angiogenic inhibitors (TNP-470 or angiostatin) or soluble VEGF receptor 1 (VEGFR1, VEGF antagonist) into the preovulatory follicle of rhesus monkeys during the spontaneous menstrual cycle revealed that VEGF antagonists impaired ovulation and attenuated subsequent luteal function, possibly by altering normal steroidogenic-nonsteroidogenic cell interactions or differentiation without dramatically changing the number of cells (Hazzard \& Stouffer 2002, Hazzard et al. $2002 a, 2002 b)$. Conversely, many studies have indicated that exogenous injection of VEGF promoted follicular development in pigs (Shimizu et al. 2003), mice (Quintana et al. 2004), and rats (Danforth et al. 2003). These studies indicate that VEGF is one of the crucial factors for follicular development in mammals.

In rodents, although exogenous administration of VEGF stimulates follicular development (Danforth et al. 2003, Quintana et al. 2004, lijima et al. 2005), it is unknown whether the follicles generated as a result of VEGF stimulation ovulate or not. Therefore, to examine whether a large number of preovulatory follicles are produced as a result of injection of VEGF gene fragments 
directly into the rat ovary and whether these follicles ovulate, we treated immature female rats with a combination of hormones and VEGF gene. Here, we report that most ovulatory follicles generated as a result of exogenous VEGF treatment ovulated. In addition, we found that injection of VEGF gene fragments directly into the ovary had an inhibitory effect on follicular atresia in immature female rats.

\section{Results}

\section{Direct ovarian injection of VEGF gene stimulates follicular development}

To examine the effect of VEGF gene injection on follicular development, we used a combination treatment consisting of hormonal therapy (eCG and hCG) and VEGF gene fragment injection. Immature female rats received direct injection of the VEGF gene fragments into their ovaries at the age of 19,21, 23, or 25 days, and were treated with eCG (20 IU) and hCG (20 IU) at the age of 26 and 28 days respectively. The maximum number of ovulated oocytes was obtained when the VEGF gene fragments were injected into the ovaries of rats at the age of 21 days (Fig. 1). In addition, histological observation showed that the cross-sectional area of ovaries with gene injection was larger than the area of those without gene injection (Fig. 2A). Moreover, the weight of the ovary was significantly increased in the gene-injected rats compared with the control rats (Fig. 2B).

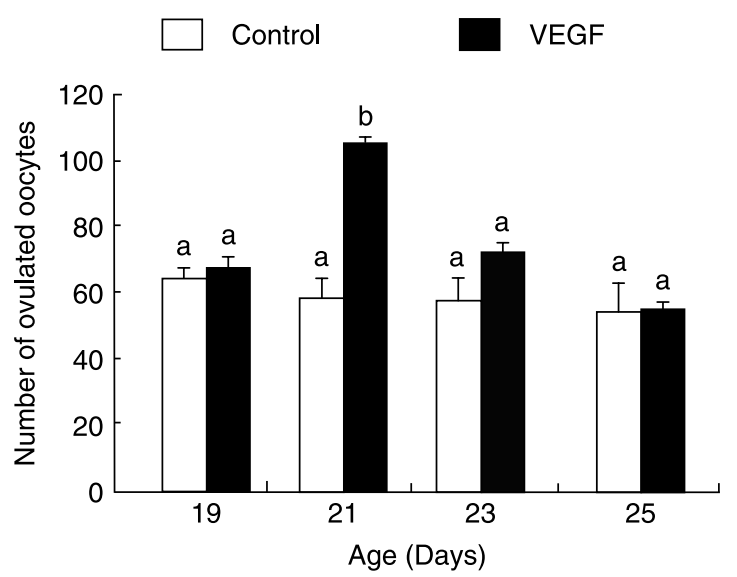

Figure 1 Effect of direct ovarian injection of VEGF gene fragments in immature rats. Direct ovarian injection of the VEGF gene fragments increased the number of ovulated oocytes in immature rats. Animals were anesthetized and VEGF gene fragments $(4 \mu \mathrm{g})$ were injected into their ovaries at 19, 21, 23, and 25 days after birth (each day: control, $n=5$; VEGF, $n=5$ ). The number of ovulated oocytes shows the number per animal. Different letters denote significantly different values $(P<0.01)$.
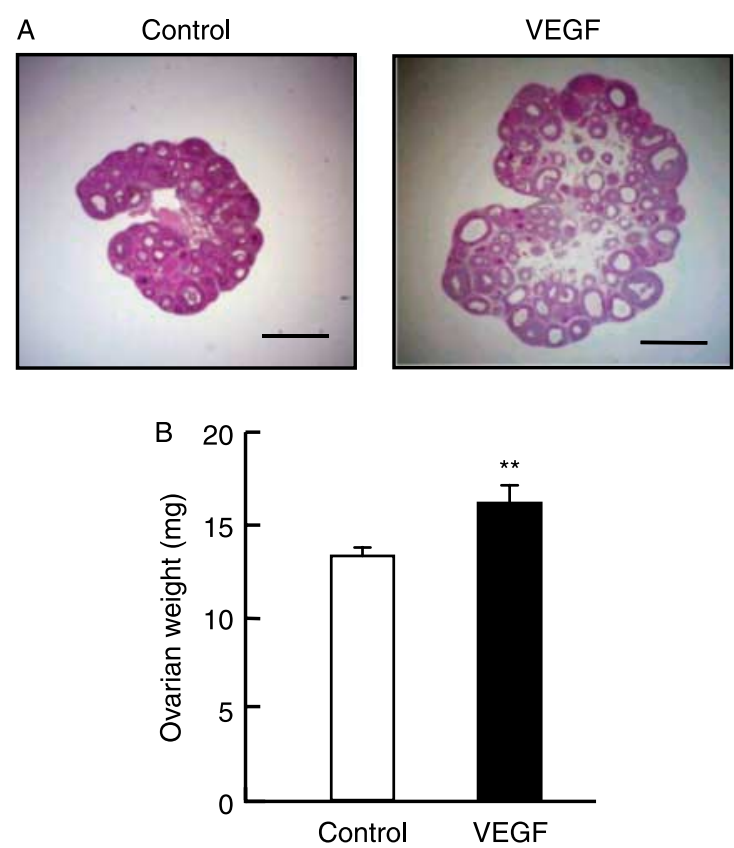

Figure 2 (A) Histological observation and (B) ovarian weight after direct ovarian injection of VEGF gene fragments. (A) Hematoxylin-eosin staining of the ovary in immature rats at 26 days of age injected with or without VEGF gene fragments. Bar indicates $500 \mu \mathrm{m}$. Each ovarian section indicates the ovary of the maximum diameter. (B) The weight of the ovaries injected with $(n=5)$ or without $(n=5)$ the VEGF gene fragments. Immature female rats were injected with VEGF gene fragments into their ovaries at the age of 21 days and the ovaries were removed at the age of 26 days. Asterisks denote significantly different values $(P<0.01)$.

\section{Promotion of follicular development by VEGF gene injection}

We examined histologically the effect of VEGF gene fragment injection on follicular development in immature female rats. VEGF gene injection at 21 days after birth stimulated the number of preantral and large follicles in the ovary at 26 days after birth (Fig. 3A). In addition, preovulatory follicles appeared as a result of VEGF gene fragment injection, and the atresia of those follicles was not observed (Fig. 3B). The preovulatory follicles and the percentage of atresia at all developmental stages in the VEGF+eCG-treated ovaries increased and decreased respectively, compared with those of the saline + eCG-treated ovaries at 28 days after birth (Fig. 4A and B).

\section{Follicular vasculature after VEGF gene injection}

We estimated the capillary density of large antral follicles ( $>350 \mu \mathrm{m}$ in diameter) in immature rats with or without VEGF gene fragment injection. Histological examination revealed that direct injection of the VEGF gene fragments markedly increased the vascular density (number of capillaries $/ \mathrm{mm}^{2}$ thecal area) around the large antral follicles (Fig. 5A). VEGF acts via its receptors, such as 
A $\square$ Control $(n=5) \quad \square$ VEGF $(n=5)$

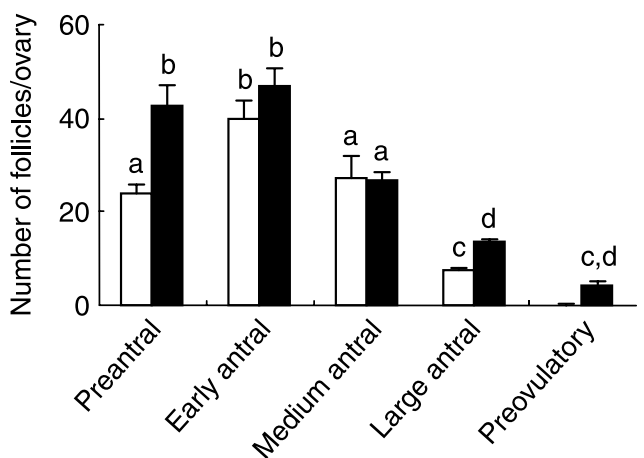

B

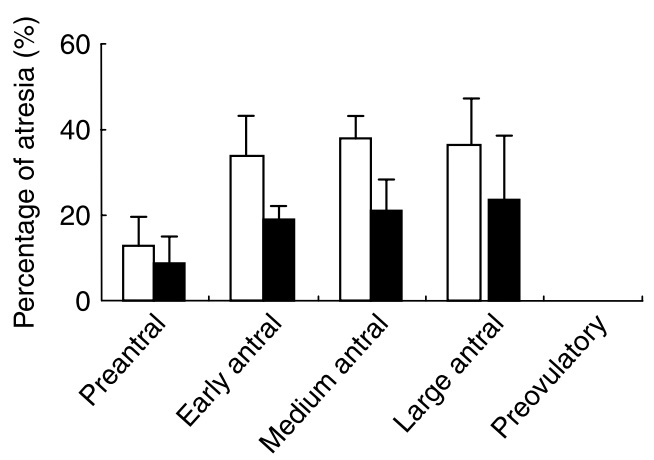

Figure 3 Changes in the follicular development in the ovaries injected with $(n=5)$ or without $(n=5)$ VEGF gene fragments. (A) The number of follicles and (B) the percentage of atretic follicles at different developmental stages in the rat ovary at 26 days after birth. Animals received direct ovarian injection of VEGF gene fragments at 21 days after birth and then the ovaries were removed at 26 days after birth. Different superscripts denote significantly different values $(P<0.05)$.

Flt-1 and Flk-1/KDR, in target cells. We found that the expression of Flk-1/KDR mRNA significantly increased in the VEGF-injected ovaries compared with those of the saline groups (Fig. 5B).

\section{Discussion}

Our data provide the first evidence that most of the ovulatory follicles generated as a result of VEGF gene fragment injection are able to ovulate. Moreover, the present study indicates that direct ovarian injection of the VEGF gene fragments stimulates the formation of vasculature in the thecal cell layer and promotes follicular development. This result is in agreement with our previous study on pigs (Shimizu et al. 2003) and other studies on mice (Quintana et al. 2004) and rats (Danforth et al. 2003).

Our findings demonstrated that the growth of the thecal vasculature of large antral follicles is induced at 5 days after direct ovarian injection of VEGF gene fragments, suggesting that the new vasculature may be formed within 5 days after VEGF injection. This increase eCG alone $(n=5)$ VEGF $+e C G(n=5)$

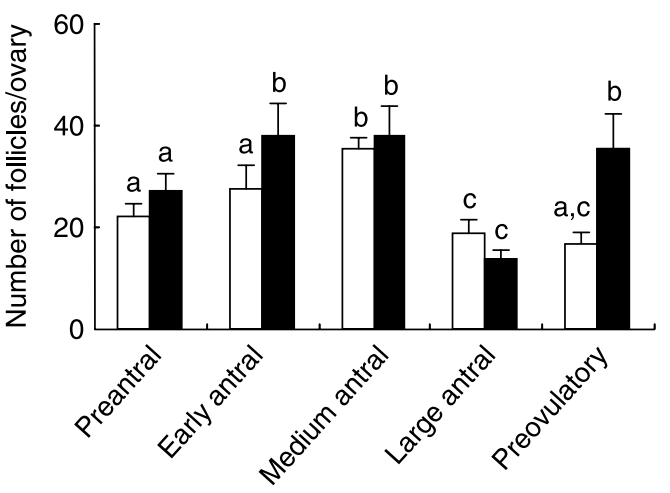

B

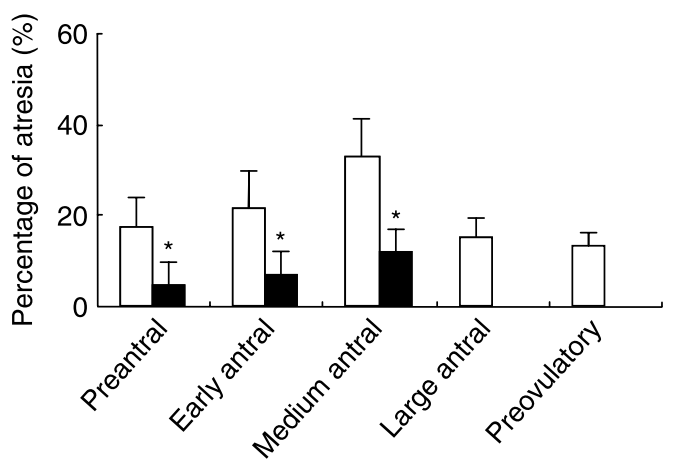

Figure 4 Changes in the follicular development in the ovaries with $(n=5)$ or without $(n=5)$ the injection of VEGF gene fragments and eCG. (A) The number of follicles and (B) the percentage of atretic follicles at different developmental stages in the rat ovary at 28 days after birth. Animals received direct ovarian injection of VEGF gene fragments at 21 days after birth, followed by i.p. administration of $20 \mathrm{IU}$ eCG at the age of 26 days, and then the ovaries were removed at 28 days after birth. Asterisks and different superscripts denote significantly different values $(P<0.05)$.

in the vascular density in the thecal cell layer may contribute to the supply of eCG into follicles and develop large antral follicles toward ovulatory stage. In fact, we found that further thecal vasculature growth was stimulated by eCG. We found that Flk-1/KDR was more strongly expressed in the ovaries treated with VEGF gene fragment injection compared with the control groups. Thus, although this result indicates the gene expression in whole ovaries, our data suggest that Flk-1/KDR may be strongly associated with the observed vascular formation around the antral follicles. In pigs, higher expression of Flt-1 was found in the VEGF+eCG-treated ovaries compared with those treated with eCG alone (Shimizu et al. 2003). The discrepancy between the findings in pigs and rats suggests that the process of vascular formation in the thecal cell layer after VEGF gene fragment injection differs between rats and pigs.

Our findings demonstrated that direct ovarian injection of VEGF gene fragments increased the number of 
A

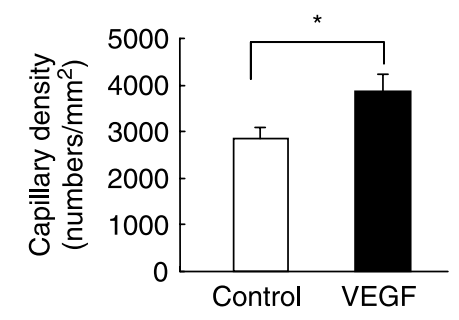

B
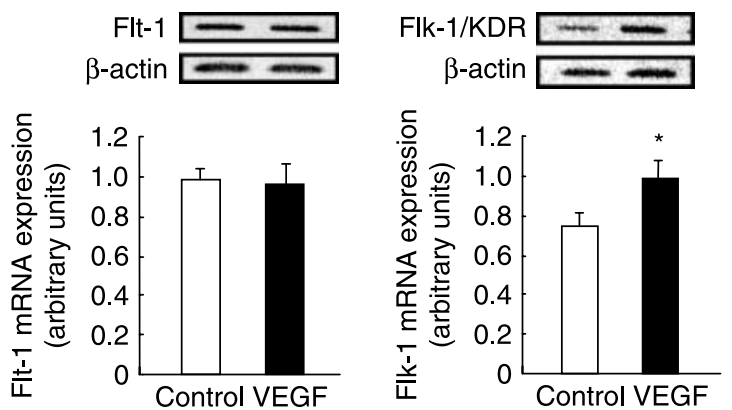

Figure 5 (A) Changes in thecal vascular density and (B) expression of Flt-1 and Flk-1 mRNAs in the ovaries after VEGF gene fragment injection. Immature rats were injected with VEGF gene fragments into their ovaries at the age of 21 days and the ovaries were removed before eCG treatment (26 days old). (A) All capillaries larger than $10 \mu \mathrm{m}$ in diameter in the section of the whole theca cell layer of antral follicles larger than $350 \mu \mathrm{m}$ in diameter were counted. (B) Expression of Flt-1 and Flk-1/KDR in the ovaries injected with $(n=5)$ or without $(n=5)$ VEGF gene fragments. The data are expressed as mean \pm s.E.M. of five animals with duplicate determination in each. Asterisks denote significantly different values $(P<0.05)$.

large antral and preantral follicles. Our data agree with the report of Danforth et al. (2003) showing that administration of VEGF into the rat ovary stimulated the development of preantral follicles. The preantral follicles have the thecal layer surrounding them (lijima et al. 2006). It has been reported in rabbits (Kanzaki et al. 1982, Kitai et al. 1985, Kikuta et al. 1991, Macchiarelli et al. 1993), cows (Yamada et al. 1994), and pigs (Jiang et al. 2002, 2004) that the single-layered capillary wreath in preantral follicles develops into a multilayered structure in the thickened theca interna layer as the follicles develop into larger and preovulatory follicles. Thus, VEGF gene fragment injection may contribute to the growth of preantral follicles by developing the thecal layer including the blood vessels to supply the nutrients. Interestingly, preovulatory follicles appeared in the ovaries after VEGF gene fragment injection, whereas such follicles did not appear in the ovaries in the control groups. A previous study showed that VEGF stimulates $\left[{ }^{3} \mathrm{H}\right]$ thymidine uptake in granulosa cells collected from medium-sized and large follicles (Grasselli et al. 2002). Therefore, VEGF may induce the development of large follicles by stimulating granulosa cell proliferation. On the other hand, in the present study, the number of early and medium antral follicles was not affected by VEGF gene fragment injection. However, we do not know why early and medium antral follicles were not affected by the VEGF gene injection.

We found that although there was no significant difference in the percentage of atretic follicles between the saline and VEGF gene fragment injection groups, the characteristics of atretic follicles changed as a result of the VEGF gene fragment injection. In particular, atretic follicles of preovulatory stage were not found. In addition, our results indicated that large antral follicles had more vasculature after VEGF gene fragment injection. Early atretic follicles can regenerate when placed in culture, suggesting that the follicle may remain in an atretic state due to a decrease in vascularity that limits access to nutrients, substrates, and tropic hormones (Moor \& Seamark 1986). Therefore, the increased formation of the thecal vascular network as a result of VEGF gene injection may contribute to the suppression of follicular atresia by supplying the nutrients and growth factors that are required for granulosa cell function. In fact, in the present study, we observed that eCG treatment resulted in a decrease in the proportion of atretic follicles in the VEGF gene fragment injection groups compared with the control groups. In general, in the mammalian ovary, apoptosis is a mechanism by which follicular atresia is induced (Guthrie et al. 1995). VEGF reduces bovine granulosa cell apoptosis in vitro (Greenaway et al. 2004). Thus, in addition to its roles in vascular formation, VEGF may act directly on granulosa cell function during follicular development. Therefore, the present study suggests that VEGF suppresses follicular atresia (granulosa cell apoptosis) and induces the production of a large number of preovulatory follicles in immature female rats.

In conclusion, our present results demonstrate that injection of VEGF gene fragments directly into the rat ovary stimulated the development of antral follicles by inducing the formation of thecal vasculature. In addition, our data provide the first evidence that most ovulatory follicles generated as a result of VEGF gene fragment injection are able to ovulate upon hCG treatment. However, the effect of VEGF gene fragment injection on the quality of the ovulated oocytes is still unknown. Therefore, in future studies, it will be necessary to examine the fertilization and development of the ovulated oocytes.

\section{Materials and Methods}

\section{Animals and preparation of VEGF gene fragments for in vivo injection}

Using a TAP Express Kit (Gene Therapy Systems Inc., San Diego, CA, USA) as previously described (Shimizu et al. 2003), porcine VEGF gene fragments (product size: VEGF120, 
$1357 \mathrm{bp}$ and VEGF164, $1489 \mathrm{bp}$ ) were transformed into a transcriptionally active PCR fragment to be used for direct injection into mammalian tissues according to the manufacturer's instructions. Briefly, custom oligos were used to produce the TAP primary fragment, which was then added to TAP Express promoter and terminator mixes. The $5^{\prime}$ - and $3^{\prime}$-custom oligos must contain 46 and 40 nucleotides respectively; of these, 26 (5'-CTG CAG GCA CCG TCG TCG ACT TAA CA- $\left.3^{\prime}\right)$ and $20\left(5^{\prime}\right.$-CAT CAA TGT ATC TTA TCA TGT CTG A-3') nucleotides comprise the $5^{\prime}$ - and $3^{\prime}$-TAP Ends respectively. The universal sequence and the other 20 (VEGF sequence; $5^{\prime}$-ATG AAC TTT CTG CTG TCT TG-3' and $5^{\prime}$-TCA CCG CCT CGG CTT GTC AC-3') nucleotides were used to make up the porcine VEGF sequence. The sequences of the VEGF genes before injection were confirmed using an ABI PRISM 310 Genetic Analyzer (Applied Biosystems, Foster City, CA, USA). Immature female Wistar-Imamichi rats were used and 60 animals were used in ovulation protocol and histological studies.

\section{Direct injection of VEGF gene into immature rat ovaries}

Animals were divided into two groups: one group received solution containing transfection reagent as a control $(n=5$ each day), while the other group received a single in situ injection of VEGF gene fragments (ovarian direct injection: $4 \mu \mathrm{g}$ DNA/1 $\mu \mathrm{l}$ reagent buffer/ovary) at the age of $19 \quad(n=5), 21 \quad(n=5)$, $23(n=5)$, or $25(n=5)$ days after birth followed by i.p. administration of $20 \mathrm{IU}$ eCG (Teikoku Zouki Pharmaceutical Co., Tokyo, Japan) at the age of 26 days. In addition, $48 \mathrm{~h}$ after eCG injection, the animals were given 20 IU hCG i.p. and the oocytes that were ovulated in both groups were recovered from the oviducts at 16-18 $\mathrm{h}$ after hCG treatment.

\section{Histological examination}

The ovaries were removed at 26 (control group, $n=5$; VEGF gene fragments-injected group, $n=5$ ) and 28 days of age (eCG alone group, $n=5$; eCG + VEGF-treated group, $n=5$ ) after VEGF gene fragment injection. The ovaries were fixed in Bouin's solution, embedded in paraffin wax, and then sectioned serially at $5 \mu \mathrm{m}$ thickness. Sequential sections were mounted and stained with hematoxylin-eosin. To avoid counting the same follicle more than once, only individual follicles having an oocyte with a nucleus were evaluated, and the size of the follicle in which the oocyte was present was measured using an ocular micrometer. Each follicle was classified as either healthy or atretic according to the absence or presence of ten pyknotic bodies in the granulosa cells of the section respectively. On the basis of their diameter, the follicles were categorized into five classes: 50-120 (preantral follicle), 121-170 (early antral follicle), 171-350 (medium antral follicle), 351-500 (large antral follicle), and $>500 \mu \mathrm{m}$ (preovulatory follicle). In addition, the capillary density of follicles $>350 \mu \mathrm{m}$ in diameter was assessed. All capillaries with diameter larger than $10 \mu \mathrm{m}$ in the sections of the whole theca interna of follicles were counted under $200 \times$ magnification $(20 \times$ objective lens and $10 \times$ ocular lens).

\section{Expression of Flt-1 and Flk-1 mRNAs}

Total cellular RNA was extracted from the ovaries (26 days after birth) at 5 days after saline or VEGF gene fragment injection with an RNeasy Mini Kit (Qiagen K.K). Semiquantitative RT-PCR was performed using Ready-To-Go RT-PCR Beads (Amersham Pharmacia Biotech Inc. Piscataway, NJ, USA), following the method recommended by the manufacturer with $100 \mathrm{ng}$ RNA. The RT reaction was carried out at $42{ }^{\circ} \mathrm{C}$ for $15 \mathrm{~min}$ and the samples were incubated at $95^{\circ} \mathrm{C}$ for $5 \mathrm{~min}$ to inactivate the reverse transcriptase and completely denature the template. Primers for Flt-1 (Miyabayashi et al. 2005) were 5'-GCT CTG TGG TTC TGC GTG GA-3' and 5'-CAT GGG ATC ACC ACA GTT TT-3'. Primers for Flk-1 (Miyabayashi et al. 2005) were 5'-GCA GCA CCT TGA CCT TGA AC-3' and $5^{\prime}$-AGG ATT GTA TTG GTC TGC CG-3'. Primers for $\beta$-actin (Miyabayashi et al. 2005) were 5'-AGC CAT GTA CGT AGC CAT- $3^{\prime}$ and $5^{\prime}$-CTC TCA GCT GTG GTG GTG AA-3' . The final cycle included a further $5 \mathrm{~min}$ at $72{ }^{\circ} \mathrm{C}$ for complete strand extension. The RT-PCR products were electrophoresed on a $2 \%$ agarose gel and visualized by ethidium bromide staining. The bands were quantified by densitometry using the NIH Image 1.63 analysis program (NIH, Bethesda, MD, USA). Expression of $\beta$-actin mRNA is not affected by growth factors or gonadotropins (Lapolt et al. 1990, Weiner \& Dias 1993). Therefore, the mRNA level for each gene in the present study was normalized on the basis of the $\beta$-actin mRNA level.

\section{Statistical analysis}

All data are presented as mean \pm s.E.M. Significant differences in the ovary weight, capillary density, and gene expression between the control and VEGF gene fragments-injected groups were analyzed using Student's $t$-test. The percentage of atretic follicles at the different developmental stages was analyzed using the $\chi^{2}$ test. The number of follicles per ovary at 26 and 28 days after birth between each treatment group was tested for significant differences using ANOVA, followed by the TukeyKramer test as a multiple comparison test. Differences were considered significant at $P<0.05$.

\section{Acknowledgements}

This work was supported by the Program for Promotion of Basic Research Activities for Innovative Biosciences and by a grant from Japan Livestock Technology Association to E S. The authors declare that there is no conflict of interest that would prejudice the impartiality of this scientific work.

\section{References}

Barboni B, Turriani M, Galeati G, Spinaci M, Bacci ML, Forni M \& Mattioli M 2000 Vascular endothelial growth factor production in growing pig antral follicles. Biology of Reproduction 63 858-864.

Celik-Ozenci C, Akkoyunlu G, Ali Kayisli U, Arici A \& Demir R 2003 Localization of vascular endothelial growth factor in the zona pellucida of developing ovarian follicle in the rat: a possible role in destiny of follicles. Histochemistry and Cell Biology 120 383-390. 
Danforth DR, Arbogast LK, Ghosh S, Dickerman A, Rofagha R \& Friedman CI 2003 Vascular endothelial growth factor stimulates preantral follicle growth in the rat ovary. Biology of Reproduction 68 1736-1741.

Grasselli F, Basini G, Bussolati S \& Tamanini C 2002 Effects of VEGF and bFGF on proliferation and production of steroids and nitric oxide in porcine granulosa cells. Reproduction of Domestic Animals 37 362-368.

Greenaway J, Connor K, Pedersen HG, Coomber BL, LaMarre J \& Petrik J 2004 Vascular endothelial growth factor and its receptor, Flk-1/KDR, are cytoprotective in the extravascular compartment of the ovarian follicle. Endocrinology 145 2896-2905.

Greenaway J, Gentry PA, Feige J, LaMarre J \& Petrik JJ 2005 Thrombospondin and vascular endothelial growth factor are cyclically expressed in an inverse pattern during bovine ovarian follicle development. Biology of Reproduction 72 1071-1078.

Guthrie HD, Grimes RW, Cooper BS \& Hammond JM 1995 Follicular atresia in pigs: measurement and physiology. Journal of Animal Science 73 2834-2844.

Hazzard TM \& Stouffer RL 2000 Angiogenesis in ovarian follicular and luteal development. Baillière's Best Practice and Research. Clinical Obstetrics and Gynaecology 14 883-900.

Hazzard TM, Rohan RM, Molskness TA, Fanton JW, D'Amato RJ \& Stouffer RL 2002a Injection of antiangiogenic agents into the macaque preovulatory follicle: disruption of corpus luteum development and function. Endocrine 17 199-206.

Hazzard TM, Xu F \& Stouffer RL $2002 b$ Injection of soluble vascular endothelial growth factor receptor 1 into the preovulatory follicle disrupts ovulation and subsequent luteal function in rhesus monkeys. Biology of Reproduction 67 1305-1312.

lijima K, Jiang JY, Shimizu T, Sasada H \& Sato E 2005 Acceleration of follicular development by administration of vascular endothelial growth factor (VEGF) protein in cycling female rats. Journal of Reproduction and Development 51 161-168.

lijima K, Tawara Y, Shimizu T, Yogo K, Sasada H \& Sato E 2006 Involvement of vascular endothelial growth factor in the formation of the thecal layer and vasculature during follicular development in the ovaries of neonatal female rats. Animal Science Journal 77 574-581.

Jiang JY, Macchiarelli G, Miyabayashi K \& Sato E 2002 Follicular microvasculature in the porcine ovary. Cell and Tissue Research $\mathbf{3 1 0}$ 93-101.

Jiang JY, Shimizu T, Sasada H, Tsang BK \& Sato E 2004 Increased ovarian follicular angiogenesis and dynamic changes of follicular vascular plexuses induced by equine chorionic gonadotropin in the gilt. Cell and Tissue Research 316 349-357.

Kanzaki H, Okamura H, Okuda Y, Takenaka A, Morimoto K \& Nishimura T 1982 Scanning electron microscopic study of rabbit ovarian follicle microvasculature using resin injection-corrosion casts. Journal of Anatomy 134 697-704.

Kikuta A, Macchiarelli G \& Murakami T 1991 Microvasculature of the ovary. In Ultrastructure of the Ovary, pp 239-254. Eds G Familiari, S Makabe \& PM Motta. Boston: Kluwer.
Kitai H, Yoshimura Y, Wright KH, Santulli R \& Wallach EE 1985 Microvasculature of preovulatory follicles: comparison of in situ and in vitro perfused rabbit ovaries following stimulation of ovulation. American Journal of Obstetrics and Gynecology 152 889-895.

LaPolt PS, Piquette GN, Soto D, Sincich C \& Hsueh AJ 1990 Regulation of inhibin messenger ribonucleic acid level by gonadotropins, growth factors, and gonadotropin-releasing hormone in cultured rat granulosa cells. Endocrinology 127 823-831.

Leung DW, Cachianes G, Kuang W, Goeddel DV \& Ferrara N 1989 Vascular endothelial growth factor is secreted angiogenic mitogen. Science $\mathbf{2 4 6}$ 1306-1309.

Macchiarelli G, Nottola SA, Vizza E, Familiari G, Kikuta A, Murakami T \& Motta PM 1993 Microvasculature of growing and atretic follicles in the rabbit ovary: a SEM study of corrosion casts. Archives of Histology and Cytology 56 1-12.

Mattioli M, Barboni B, Turriani M, Galeati G, Zannoni A, Castellani G, Berardinelli P \& Scapolo PA 2001 Follicle activation involves vascular endothelial growth factor production and increased blood vessel extension. Biology of Reproduction 65 1014-1019.

Miyabayashi K, Shimizu T, Kawauchi C, Sasada H \& Sato E 2005 Changes of mRNA expression of vascular endothelial growth factor, angiopoietins and their receptors during the periovulatory period in eCG/hCGtreated immature female rats. Journal of Experimental Zoology 303A 590-597.

Moor RM \& Seamark RF 1986 Cell signaling, permeability, and microvasculatory changes during antral follicle development in mammals. Journal of Dairy Science 69 927-943.

Quintana R, Kopcow L, Sueldo C, Marconi G, Rueda NG \& Baranao RI 2004 Direct injection of vascular endothelial growth factor into the ovary of mice promotes follicular development. Fertility and Sterility 82 1101-1105.

Shimizu T, Jiang JY, Sasada H \& Sato E 2002 Changes of messenger RNA expression of angiogenic factors and related receptors during follicular development in gilts. Biology of Reproduction 67 1846-1852.

Shimizu T, Jiang JY, lijima K, Miyabayashi K, Ogawa Y, Sasada H \& Sato E 2003 Induction of follicular development by direct single injection of vascular endothelial growth factor gene fragments into the ovary of miniature gilts. Biology of Reproduction 69 1388-1393.

Weiner KX \& Dias JA 1993 Regulation of ovarian ornithin decarboxylase activity and its mRNA by gonadotropins in the immature rat. Molecular and Cellular Endocrinology 92 195-199.

Yamada O, Abe M, Takehara K, Iwasa K, Hiraga T \& Hiratsuka T 1994 Microvasculature of mature bovine follicles and its changes with ovulation. Journal of Reproduction and Development 40 307-315.

Received 13 June 2007

First decision 14 June 2007

Revised manuscript received 10 July 2007

Accepted 13 August 2007 